\title{
Improvement of Calculation Accuracy in the Electron Monte Carlo Algorithm with Optional Air Profile Measurements
}

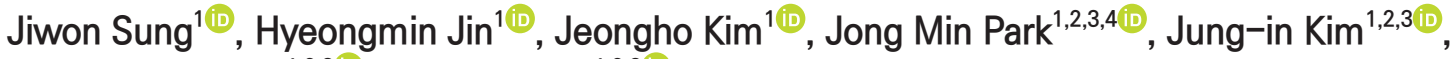 \\ Chang Heon Choi ${ }^{1,2,3[i]}$, Minsoo Chun ${ }^{1,2,3}$ (i] \\ 'Department of Radiation Oncology, Seoul National University Hospital, ${ }^{2}$ Biomedical Research Institute, Seoul National University Hospital, \\ ${ }^{3}$ Institute of Radiation Medicine, Seoul National University Medical Research Center, ${ }^{4}$ Department of Radiation Oncology, Seoul National \\ University College of Medicine, Seoul, Korea
}

Received 1 September 2020 Revised 23 October 2020

Accepted 9 November 2020

Corresponding author

Minsoo Chun

(ms1236@snu.ac.kr)

Tel: 82-2-2072-4160

Fax: 82-2-765-3317
Purpose: In this study, the accuracies of electron Monte Carlo (eMC) calculation algorithms were evaluated to determine whether electron beams were modeled by optional air profiles (APs) designed for each applicator size.

Methods: Electron beams with the energies of 6, 9, 12, and $16 \mathrm{MeV}$ for VitalBeam (Varian Medical System, Palo Alto, CA, USA) and 6, 9, 12, 16, and $20 \mathrm{MeV}$ for Clinac iX (Varian Medical System) were used. Optional APs were measured at the source-to-detector distance of $95 \mathrm{~cm}$ with jaw openings appropriate for each machine, electron beam energy, and applicator size. The measured optional APs were postprocessed and converted into the w2CAD format. Then, the electron beams were modeled and calculated with and without optional APs. Measured profiles, percentage depth doses, penumbras with respect to each machine, and energy were compared to calculated dose distributions.

Results: For VitalBeam, the profile differences between the measurement and calculation were reduced by $0.35 \%, 0.15 \%, 0.14 \%$, and $0.38 \%$ at $6,9,12$, and $16 \mathrm{MeV}$, respectively, when the beams were modeled with APs. For Clinac iX, the differences were decreased by $0.16 \%,-0.31 \%$, $0.94 \%, 0.42 \%$, and $0.74 \%$, at $6,9,12,16$, and $20 \mathrm{MeV}$, respectively, with the insertion of APs. Of note, no significant improvements in penumbra and percentage depth dose were observed, although the beam models were configured with APs.

Conclusions: The accuracy of the eMC calculation can be improved in profiles when electron beams are modeled with optional APs.

Keywords: Electron Monte Carlo, Air profile measurement, Electron applicator, Beam configuration, Eclipse treatment planning system

\section{Introduction}

Monte Carlo (MC) simulation is one of the most accurate methods for calculating dose distributions, particularly in heterogeneous tissues, where the effects of electron transport cannot be accurately modeled with conventional deterministic dose calculation algorithms [1,2]. Despite the accuracy of the MC simulation, large calculation times have limited its application in clinical routines [3]. Recently, with an increase in the computer processing speed, the

Copyright (c) 2020 Korean Society of Medical Physics

@This is an Open-Access article distributed under the terms of the Creative Commons Attribution Non-Commercial License (http://creativecommons.org/licenses/by$\mathrm{nc} / 4.0$ ) which permits unrestricted non-commercial use, distribution, and reproduction in any medium, provided the original work is properly cited. 
a

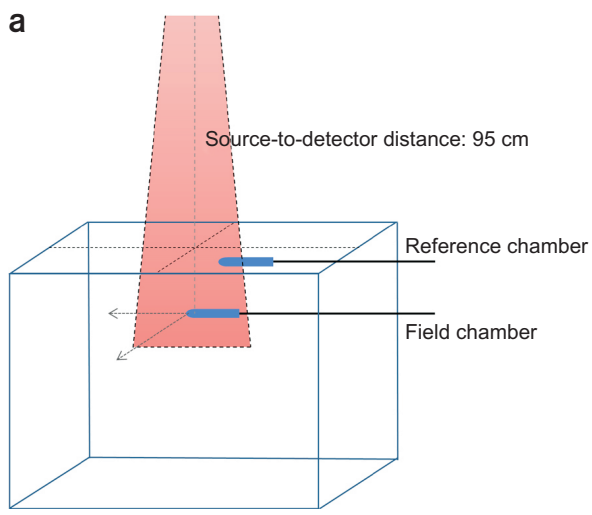

b

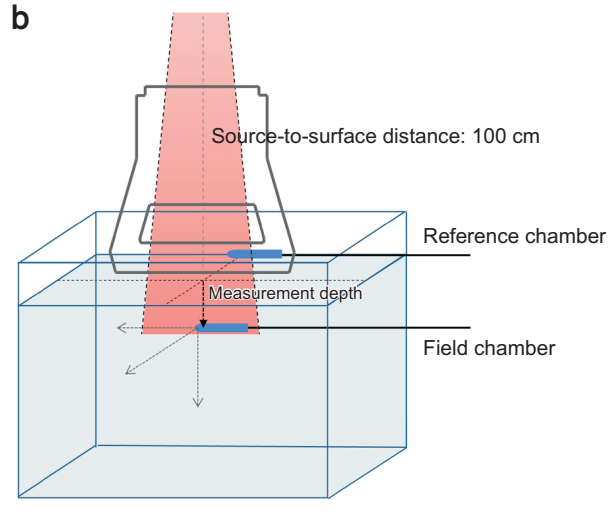

Fig. 1. Schematic diagrams of the measurement setup for (a) optional air profiles and (b) verifications. performance of the MC algorithm has been improved, and the MC dose calculation became acceptable for clinical treatment planning with electron beams [4-6]. The electron Monte Carlo (eMC) algorithm is based on the macro MC method for the electron beam in the Eclipse treatment planning system (Varian Medical System, Palo Alto, CA, USA) [7-9]. The eMC algorithm increases the calculation speed while preserving the calculation accuracy in heterogeneous internal parts of patients, such as the lung and oral cavity $[10,11]$.

However, some limitations hinder the use of the eMC algorithm. Several studies have demonstrated discrepancies between measurements and calculations, particularly for lower electron energies, smaller applicator sizes, and larger source-to-surface distances (SSDs) [10,12-14]. In the buildup region at $4 \mathrm{MeV}$, the eMC algorithm produces up to $5.4 \%$ lower absorbed dose than that of the full MC model. In the penumbra and out-of-field regions in water, the eMC calculation underestimates the absorbed doses by up to $3.3 \%$, compared to the full MC model [15]. The eMC calculation overestimates the absorbed dose toward the shoulder region with a $25 \times 25 \mathrm{~cm}^{2}$ applicator at increased SSDs [15].

Varian Medical System has announced that the beam configuration with air profiles (APs) can improve the calculation accuracy with AP measurements at the sourceto-detector distance (SDD) of $95 \mathrm{~cm}$ where the collimator jaws open appropriately for each machine, energy, and applicator [16]. The purpose of this study is to evaluate the performances of the eMC algorithm with the insertion of APs in terms of profile, percentage depth dose (PDD), and penumbra.

\section{Materials and Methods}

\section{AP measurements and beam configuration}

This study was conducted within electron energy ranges used at the author's hospital. The electron energies of 6, 9, 12, and $16 \mathrm{MeV}$ for VitalBeam (Varian Medical System) and $6,9,12,16$, and $20 \mathrm{MeV}$ for the Clinac iX linear accelerator were used in this study. A Blue Phantom² scanning system (IBA Dosimetry GmbH, Schwarzenbruck, Germany) and two CC13 cylindrical ionization chambers (IBA Dosimetry $\mathrm{GmbH}$ ) were used to acquire APs. The field chamber was placed at the SDD of $95 \mathrm{~cm}$ in air to measure APs. To remove pulsed beam fluctuations, the reference chamber was placed so that the active volume could be fully covered by the corresponding field sizes [17]. The measurement setup for APs is illustrated in Fig. 1a. The in-line and cross-line APs were measured, in which jaw positions were opened according to the applicator without an attached applicator [16]. The jaw sizes for each machine, energy, and applicator size are shown in Table 1. The measured APs were postprocessed by smoothing and rescaling; then, they were converted into the w2CAD format to register the measured beam data to Eclipse. The final APs with the w2CAD format were imported and configured according to the Eclipse reference guide document [16].

\section{Measurement of the reference dose distribution}

To evaluate the accuracy of the eMC algorithm with APs, the dose profiles and PDD curves were measured using the Blue Phantom² scanning system. The measurement setup 
Table 1. Jaw openings according to the applicator sizes and energies for VitalBeam and Clinac iX

\begin{tabular}{|c|c|c|c|c|c|c|c|c|c|}
\hline \multirow{3}{*}{$\begin{array}{l}\text { Applicator } \\
\text { size }\left(\mathrm{cm}^{2}\right)\end{array}$} & \multicolumn{9}{|c|}{ Jaw openings $\left(\mathrm{cm}^{2}\right)$} \\
\hline & \multicolumn{4}{|c|}{ VitalBeam } & \multicolumn{5}{|c|}{ Clinac iX } \\
\hline & $6 \mathrm{MeV}$ & $9 \mathrm{MeV}$ & $12 \mathrm{MeV}$ & $16 \mathrm{MeV}$ & $6 \mathrm{MeV}$ & $9 \mathrm{MeV}$ & $12 \mathrm{MeV}$ & $16 \mathrm{MeV}$ & $20 \mathrm{MeV}$ \\
\hline $6 \times 6$ & $20 \times 20$ & $20 \times 20$ & $11 \times 11$ & $11 \times 11$ & $20 \times 20$ & $20 \times 20$ & $11 \times 11$ & $11 \times 11$ & $11 \times 11$ \\
\hline $10 \times 10$ & $22 \times 22$ & $20 \times 20$ & $15 \times 15$ & $15 \times 15$ & $20 \times 20$ & $20 \times 20$ & $14 \times 14$ & $14 \times 14$ & $14 \times 14$ \\
\hline $15 \times 15$ & $22 \times 22$ & $20 \times 20$ & $19 \times 19$ & $18 \times 18$ & $20 \times 20$ & $20 \times 20$ & $17 \times 17$ & $17 \times 17$ & $17 \times 17$ \\
\hline $20 \times 20$ & $27 \times 27$ & $25 \times 25$ & $25 \times 25$ & $23 \times 23$ & $25 \times 25$ & $25 \times 25$ & $25 \times 25$ & $23 \times 23$ & $22 \times 22$ \\
\hline $25 \times 25$ & $32 \times 32$ & $30 \times 30$ & $30 \times 30$ & $28 \times 28$ & $30 \times 30$ & $30 \times 30$ & $30 \times 30$ & $28 \times 28$ & $27 \times 27$ \\
\hline
\end{tabular}

for reference dose distributions is shown in Fig. 1b. Blue Phantom ${ }^{2}$ was positioned with the SSD of $100 \mathrm{~cm}$. Similar to the measurement of APs, to remove pulsed beam fluctuations, the reference chamber was placed where the active volume could be fully covered by the corresponding field sizes [17]. For VitalBeam, the profiles were measured at the depth of 1.18, 1.58, 2.20 and $2.90 \mathrm{~cm}$ at 6, 9, 12, and $16 \mathrm{MeV}$, respectively. For Clinac iX, the measurement depths were $1.00,1.40,2.00,2.70$, and $3.30 \mathrm{~cm}$ at $6,9,12,16$, and $20 \mathrm{MeV}$, respectively. The depths were shifted upward by $0.15 \mathrm{~cm}$, considering the effective measurement point, with an A25 applicator $\left(25 \times 25 \mathrm{~cm}^{2}\right)$ and normalized to $100 \%$ at the center of the profiles. The PDD curves were measured with an A10 applicator $\left(10 \times 10 \mathrm{~cm}^{2}\right)$ and normalized to $100 \%$ at the depth of the maximum dose.

\section{3. eMC calculation}

The dose distributions were calculated in a $40 \times 40 \times 40 \mathrm{~cm}^{3}$ virtual water phantom using beam models with and without APs. The eMC calculation options used in this study are listed in Table 2. For the number of particle histories option, the zero value means that the eMC calculation uses as many particles as required to reach the statistical uncertainty set in statistical uncertainty option. The random generator seed number is defined as the random number sequence used in the particle generator. If the seed value is set to 0 , the EMC algorithm uses a randomly selected seed. The dose threshold for uncertainty was defined as the dose threshold to calculate statistical uncertainty [16]. The dose distributions with A25 applicators were exported in the coronal plane and normalized to $100 \%$ at the center of the profile using the RIT software (Radiological Imaging Technology,
Table 2. Electron Monte Carlo (eMC) calculation parameters used in this study

\begin{tabular}{lc}
\hline \multicolumn{1}{c}{ Calculation parameter } & Value \\
\hline Statistical uncertainty & 1 \\
Statistical uncertainty limit & 3 \\
Calculation grid size in cm & 0.25 \\
Random generator seed number & 0 \\
Number of particle histories & 0 \\
Dose threshold for uncertainty & 50 \\
Smoothing method & Three-dimensional Gaussian \\
Smoothing level & Medium \\
\hline
\end{tabular}

Inc., Colorado Springs, CO, USA). The absolute differences between calculated and measured dose distributions were averaged in the range of -10 to $10 \mathrm{~cm}$ to represent the discrepancies between the models with and without APs. The PDDs with A10 applicators were obtained and normalized to $100 \%$ at the depth of the dose maximum. Considering the effective measurement point, the comparison between the calculated and measured PDDs was started from the depth of $0.15 \mathrm{~cm}$. Both calculated and measured penumbras were obtained by averaging the distances of the right and left sides between $20 \%$ and $90 \%$ of the central axis dose in the dose profiles [18].

\section{Results}

The discrepancies in the profiles between the measurements and calculations for VitalBeam are summarized in Table 3, while the sample distributions at 6 and $16 \mathrm{MeV}$ are shown in Fig. 2. The accuracy of calculated profile was improved by $0.35 \%, 0.15 \%, 0.14 \%$, and $0.38 \%$ on average at $6,9,12$, and $16 \mathrm{MeV}$, respectively, when the beams were modeled with APs. For all energies, the calculations with 
Table 3. Improvements between measurements and calculations for VitalBeam and Clinac iX

\begin{tabular}{|c|c|c|c|c|c|c|c|c|c|}
\hline \multirow{3}{*}{$\begin{array}{l}\text { Energy } \\
(\mathrm{MeV})\end{array}$} & \multirow{3}{*}{ Direction } & \multicolumn{4}{|c|}{ VitalBeam } & \multicolumn{4}{|c|}{ Clinac iX } \\
\hline & & \multirow{2}{*}{$\begin{array}{c}\text { Measurement } \\
\text { depth }(\mathrm{cm})\end{array}$} & \multicolumn{2}{|c|}{$\begin{array}{c}\text { Calculation- } \\
\text { measurement* }(\%)\end{array}$} & \multirow{2}{*}{$\begin{array}{c}\text { Improvement } \\
(\%)\end{array}$} & \multirow{2}{*}{$\begin{array}{c}\text { Measurement } \\
\text { depth }(\mathrm{cm})\end{array}$} & \multicolumn{2}{|c|}{$\begin{array}{c}\text { Calculation- } \\
\text { measurement* }(\%)\end{array}$} & \multirow{2}{*}{$\begin{array}{c}\text { Improvement } \\
(\%)\end{array}$} \\
\hline & & & Without AP & With AP & & & Without AP & With AP & \\
\hline \multirow[t]{2}{*}{6} & In-line & 1.18 & 1.08 & 0.69 & 0.38 & 1.00 & 0.65 & 0.45 & 0.20 \\
\hline & Cross-line & & 1.07 & 0.75 & 0.32 & & 0.48 & 0.37 & 0.11 \\
\hline \multirow[t]{2}{*}{9} & In-line & 1.58 & 0.91 & 0.67 & 0.24 & 1.40 & 0.23 & 0.40 & -0.17 \\
\hline & Cross-line & & 0.76 & 0.71 & 0.05 & & 0.15 & 0.60 & -0.45 \\
\hline \multirow[t]{2}{*}{12} & In-line & 2.20 & 0.32 & 0.12 & 0.20 & 2.00 & 1.16 & 0.21 & 0.95 \\
\hline & Cross-line & & 0.19 & 0.11 & 0.08 & & 1.20 & 0.28 & 0.92 \\
\hline \multirow[t]{2}{*}{16} & In-line & 2.90 & 0.96 & 0.46 & 0.50 & 2.70 & 0.69 & 0.22 & 0.47 \\
\hline & Cross-line & & 0.66 & 0.40 & 0.26 & & 0.95 & 0.59 & 0.36 \\
\hline \multirow[t]{2}{*}{20} & In-line & - & - & - & - & 3.30 & 1.31 & 0.45 & 0.86 \\
\hline & Cross-line & & - & - & - & & 1.07 & 0.46 & 0.61 \\
\hline
\end{tabular}

AP, air profile; -, no data.

*Calculation-measurement: average value of differences between the calculated and measured profiles in the range of -10 to $10 \mathrm{~cm}$.
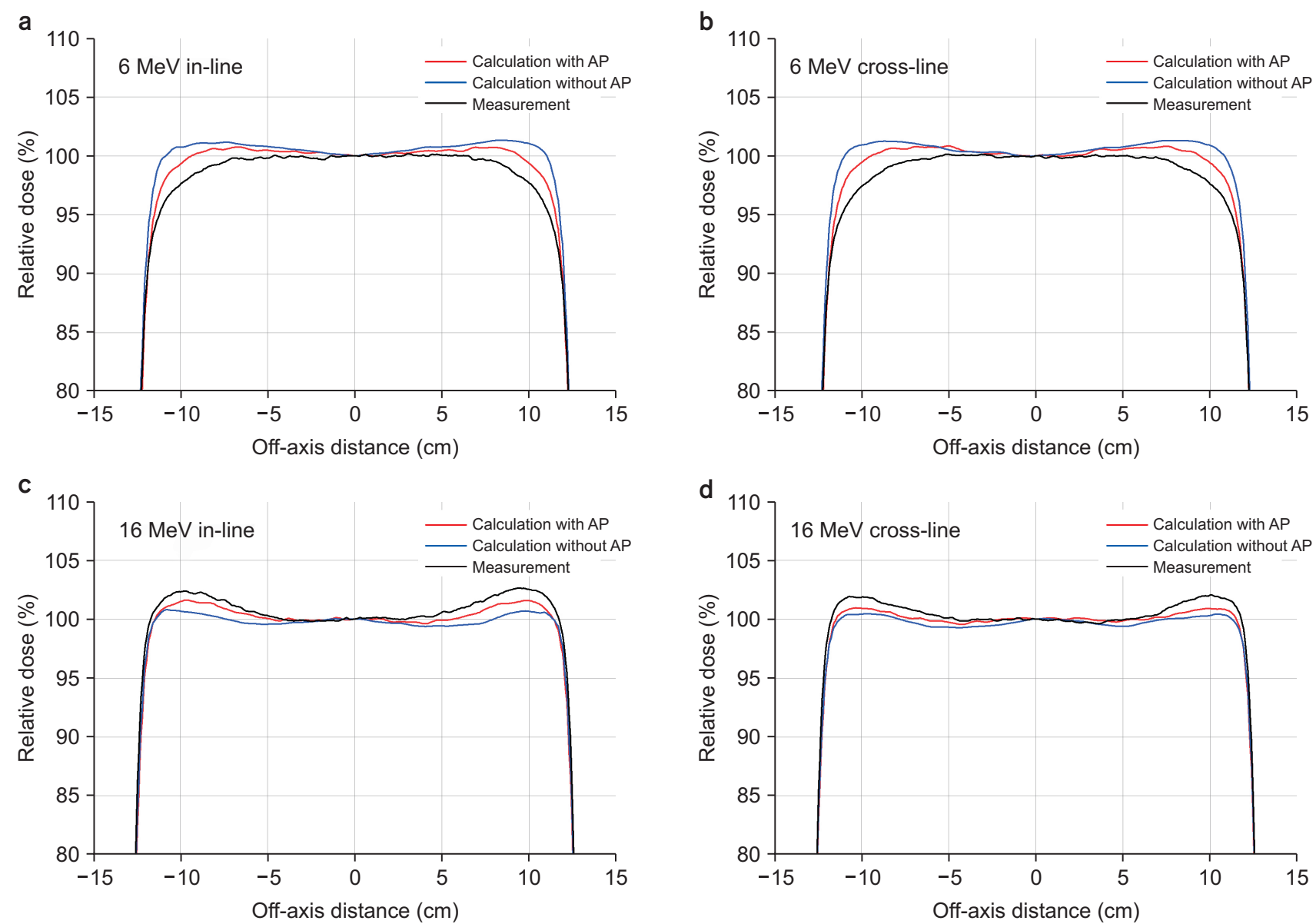

Fig. 2. Measured and calculated profiles with and without air profiles (APs) for VitalBeam: (a, c) in-line and (b, d) cross-line profiles at 6 and $16 \mathrm{MeV}$, respectively. 

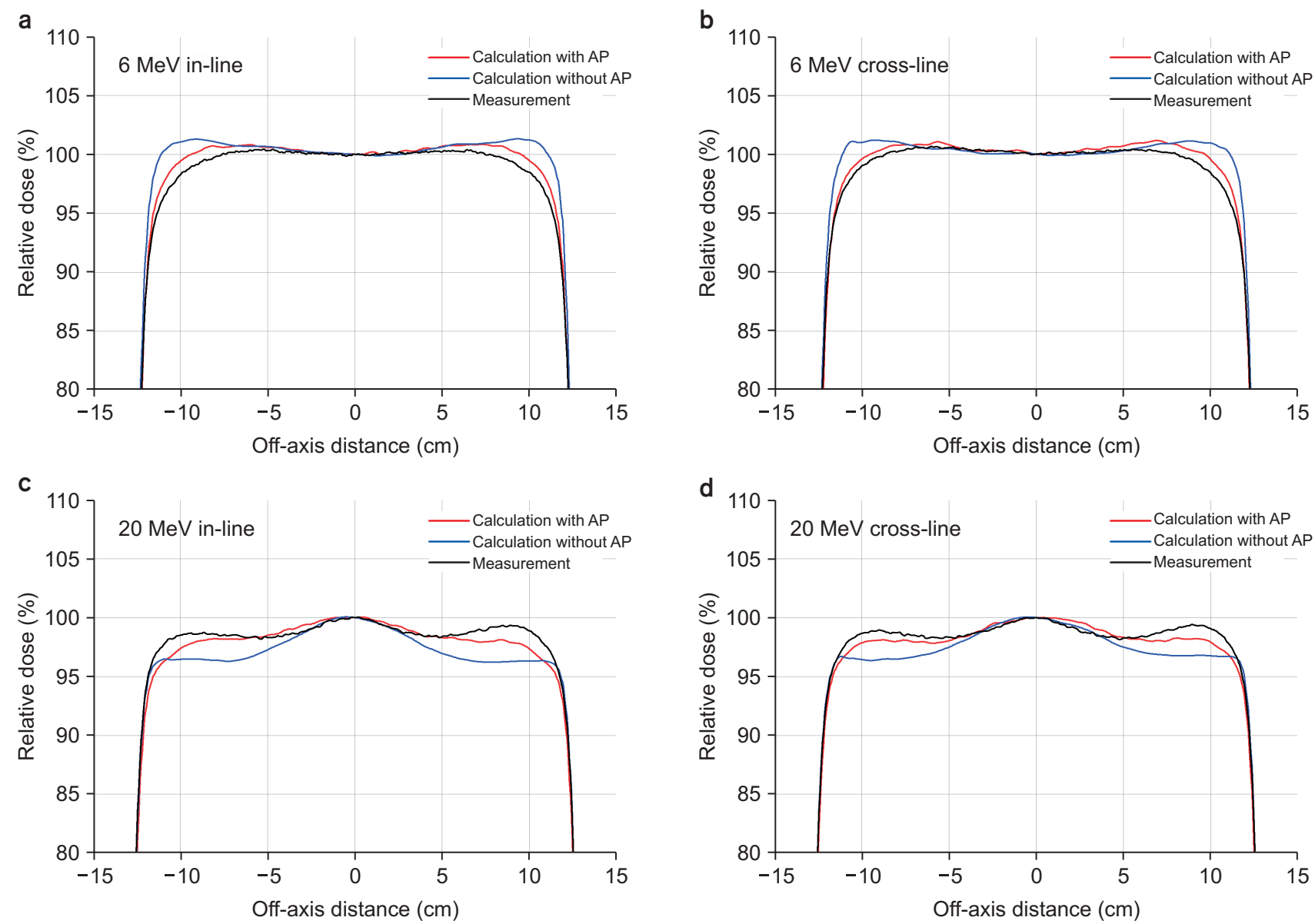

Fig. 3. Measured and calculated profiles with and without air profiles (APs) for Clinac iX: (a, c) in-line and (b, d) cross-line profiles at 6 and $20 \mathrm{MeV}$, respectively.

APs improved the accuracy up to $0.5 \%$. Although the calculations with APs still exhibited over- and under-estimation tendencies, the discrepancies were reduced at both low and high energies. The discrepancies in the profiles between the measurements and calculations for Clinac iX are summarized in Table 3, while the sample distributions at 6 and 20 $\mathrm{MeV}$ are shown in Fig. 3. The discrepancies were reduced by $0.16 \%,-0.31 \%, 0.94 \%, 0.42 \%$, and $0.74 \%$ on average at 6 , $9,12,16$, and $20 \mathrm{MeV}$, respectively, with the insertion of APs. Except at $9 \mathrm{MeV}$, the calculations with APs improved the accuracy up to $0.95 \%$. The calculations with APs at $9 \mathrm{MeV}$ had lower accuracy than those without APs of $0.17 \%$ and $0.45 \%$ for the in-line and cross-line profiles, respectively.

The penumbras are shown in Table 4. For Clinac iX, the penumbra difference between the measurement and calculation without AP was up to $0.10 \mathrm{~cm}$, while that with AP was up to $0.15 \mathrm{~cm}$. For VitalBeam, the maximum difference between the measurement and calculation was $0.25 \mathrm{~cm}$ for both cases. Of note, no significant change in the difference between the measured and calculated penumbras was observed between the cases with and without AP. Table 5 shows the maximum and average difference between the PDD measurement and calculation with or without APs, while the sample distributions at low (6 MeV) and high (16 and $20 \mathrm{MeV}$ ) energies are shown in Fig. 4. For VitalBeam, although the measured PDDs exhibited differences up to $6.09 \%, 2.99 \%, 1.71 \%$, and $1.45 \%$ without APs, those with APs were $5.68 \%, 3.02 \%, 1.95 \%$, and $1.13 \%$ at $6,9,12$, and $16 \mathrm{MeV}$, respectively. For Clinac iX, the measured PDDs exhibited differences up to $5.07 \%, 2.82 \%, 1.72 \%, 1.74 \%$, and $0.82 \%$ without APs, while those with APs were $5.24 \%$, 3.03\%, 1.50\%, $1.29 \%$, and $1.00 \%$ at $6,9,12,16$, and $20 \mathrm{MeV}$, respectively. Large discrepancies were usually observed in the buildup region, particularly at low energies. This could not be im- 
Table 4. Comparison between the measurements and calculations for the penumbras for VitalBeam and Clinac iX

\begin{tabular}{|c|c|c|c|c|c|c|c|c|c|}
\hline \multirow{3}{*}{$\begin{array}{l}\text { Energy } \\
(\mathrm{MeV})\end{array}$} & \multirow{3}{*}{ Direction } & \multicolumn{4}{|c|}{ VitalBeam } & \multicolumn{4}{|c|}{ Clinac iX } \\
\hline & & \multirow{2}{*}{$\begin{array}{c}\text { Measurement } \\
\text { depth }(\mathrm{cm})\end{array}$} & \multicolumn{2}{|c|}{ Calculation $(\mathrm{cm})$} & \multirow{2}{*}{$\begin{array}{l}\text { Measurement } \\
(\mathrm{cm})\end{array}$} & \multirow{2}{*}{$\begin{array}{c}\text { Measurement } \\
\text { depth }(\mathrm{cm})\end{array}$} & \multicolumn{2}{|c|}{ Calculation $(\mathrm{cm})$} & \multirow{2}{*}{$\begin{array}{l}\text { Measurement } \\
(\mathrm{cm})\end{array}$} \\
\hline & & & Without AP & With AP & & & Without AP & With AP & \\
\hline \multirow[t]{2}{*}{6} & In-line & 1.18 & 1.30 & 1.38 & 1.33 & 1.00 & 1.20 & 1.30 & 1.30 \\
\hline & Cross-line & & 1.30 & 1.35 & 1.30 & & 1.20 & 1.30 & 1.28 \\
\hline \multirow[t]{2}{*}{9} & In-line & 1.58 & 1.10 & 1.10 & 0.98 & 1.40 & 1.15 & 1.18 & 1.15 \\
\hline & Cross-line & & 1.05 & 1.10 & 0.95 & & 1.10 & 1.15 & 1.05 \\
\hline \multirow[t]{2}{*}{12} & In-line & 2.20 & 1.15 & 1.15 & 0.95 & 2.00 & 1.13 & 1.18 & 1.05 \\
\hline & Cross-line & & 1.15 & 1.15 & 0.95 & & 1.15 & 1.10 & 1.08 \\
\hline \multirow[t]{2}{*}{16} & In-line & 2.90 & 1.25 & 1.28 & 1.03 & 2.70 & 1.18 & 1.23 & 1.13 \\
\hline & Cross-line & & 1.25 & 1.25 & 1.00 & & 1.20 & 1.20 & 1.15 \\
\hline \multirow[t]{2}{*}{20} & In-line & - & - & - & - & 3.30 & 1.33 & 1.43 & 1.28 \\
\hline & Cross-line & & - & - & - & & 1.30 & 1.40 & 1.30 \\
\hline
\end{tabular}

AP, air profile; -, no data.

Table 5. Comparison of the mean and maximum differences in PDDs between the measurements and calculations for VitalBeam and Clinac iX

\begin{tabular}{|c|c|c|c|c|c|c|c|c|}
\hline \multirow{3}{*}{$\begin{array}{l}\text { Energy } \\
(\mathrm{MeV})\end{array}$} & \multicolumn{4}{|c|}{ VitalBeam } & \multicolumn{4}{|c|}{ Clinac iX } \\
\hline & \multicolumn{2}{|c|}{ With AP } & \multicolumn{2}{|c|}{ Without AP } & \multicolumn{2}{|c|}{ With AP } & \multicolumn{2}{|c|}{ Without AP } \\
\hline & Mean (\%) & Maximum (\%) & Mean (\%) & Maximum (\%) & Mean (\%) & Maximum (\%) & Mean (\%) & Maximum (\%) \\
\hline 6 & 1.45 & 5.68 & 1.52 & 6.09 & 0.64 & 5.24 & 0.62 & 5.07 \\
\hline 9 & 1.28 & 3.02 & 1.35 & 2.99 & 0.50 & 3.03 & 0.47 & 2.82 \\
\hline 12 & 0.94 & 1.95 & 0.84 & 1.71 & 0.29 & 1.50 & 0.36 & 1.72 \\
\hline 16 & 0.48 & 1.13 & 0.42 & 1.45 & 0.33 & 1.29 & 0.55 & 1.74 \\
\hline 20 & - & - & - & - & 0.16 & 1.00 & 0.22 & 0.82 \\
\hline
\end{tabular}

PDD, percentage depth dose; AP, air profile; -, no data.

proved even using eMC models with APs.

\section{Discussion}

In this study, the accuracy of the eMC calculation was evaluated when the electron beams were modeled with optional APs in the treatment planning system. The accuracies of profiles were improved by modeling the electron beam with optional APs. The differences could be minimized when the electron beams were modeled by adding optional APs. Specifically, this reduction was considerable in the shoulder regions where the profiles were near the dose falloff. According to the manufacturer's document, optional APs could construct two-dimensional fluences in beam modeling. If optional AP was not measured, the radially symmetric fluences from the open field APs $\left(40 \times 40 \mathrm{~cm}^{2}\right)$ are used for dose calculation. Because the actual fluences for each applicator size differ from those of open fields, the jaw size-specific measurements can improve the accuracies. The dose calculation accuracy for a given applicator can be improved by constructing a two-dimensional fluence that is appropriate for each applicator. Otherwise, the symmetric fluence in air with a $40 \times 40 \mathrm{~cm}^{2}$ open field can be applied for all applicators during the dose calculation $[14,16]$. To consider these properties, Varian Medical System recommends to include optional APs for the electron beam configuration [16].

Although no significant improvements in PDDs and penumbras were observed when eMCs were modeled with optional APs, the accuracies of profiles were improved overall. The PDD differences between measurement and eMC calculation have been reported in many studies $[4,19,20]$, although electron beams have been modeled without optional APs. For $4 \mathrm{MeV}$, the eMC algorithm produced up to $5.4 \%$ 

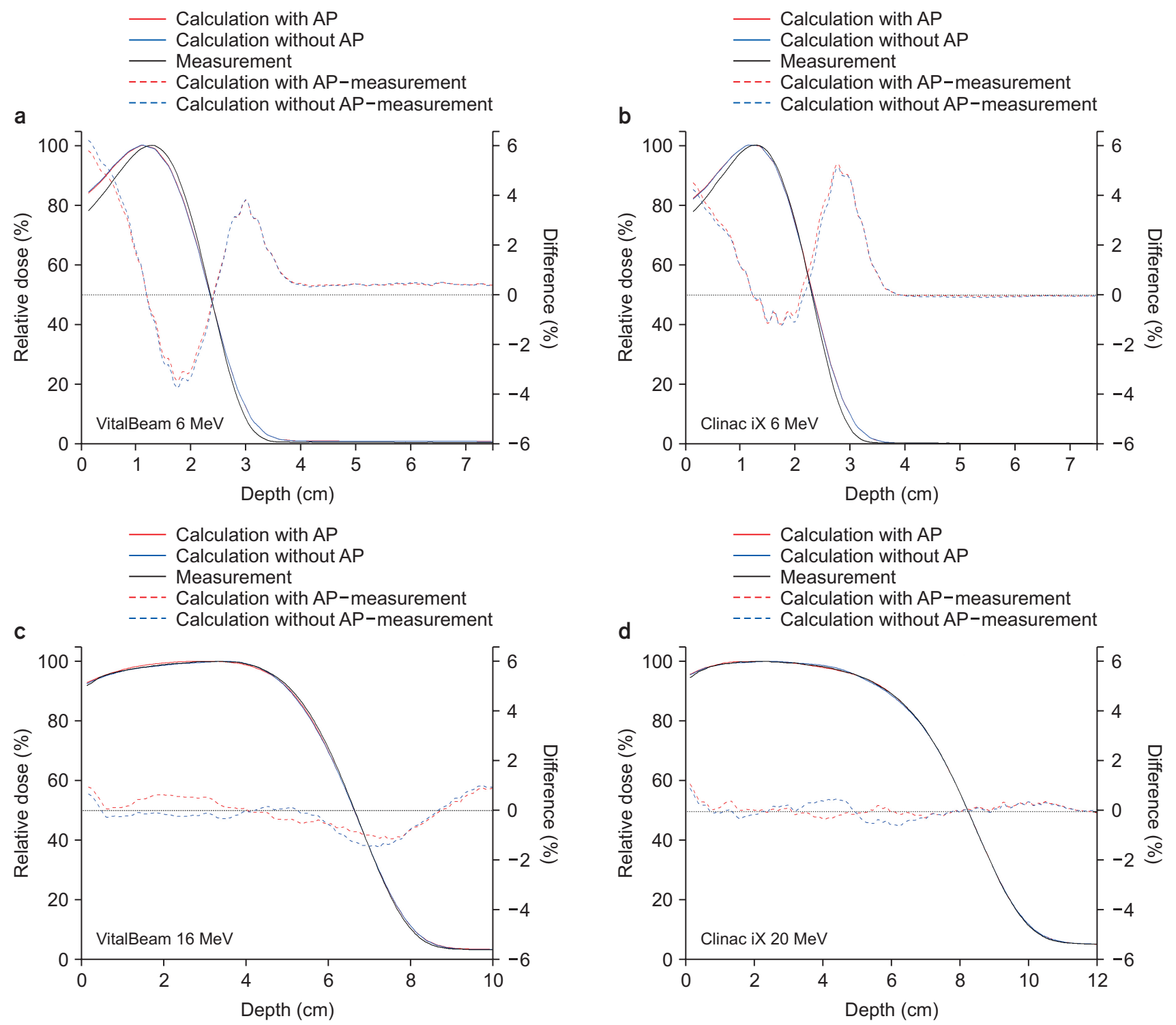

Fig. 4. Comparison of the measured and calculated percentage depth dose (PDD) curves with and without air profiles (Aps) and corresponding dose differences at (a) 6 and (c) $16 \mathrm{MeV}$ for VitalBeam and at (b) 6 and (d) $20 \mathrm{MeV}$ for Clinac iX. Calculation with APmeasurement, difference between the calculation with AP and measurement value; Calculation without AP-measurement, difference between the calculation without AP and measurement value.

lower doses than those of the full MC model in the buildup region and over-estimated the dose by up to $5.2 \%$ beyond the depth of the dose maximum, particularly at the end of the electron range [15]. This result could not be improved even with APs. The eMC algorithm largely underestimated the doses near the surface and overestimated them near the end point of the fall-off region compared to the measurement. Because this behavior was emphasized at low energies, the treatment plan should be carefully designed at low electron energy and prescription depth in the superficial region. It is necessary to improve the eMC algorithm to improve the calculation accuracy at low energy.

\section{Conclusions}

The accuracy of eMC calculation can be improved in dose profiles when the electron beams are modeled with optional APs. 


\section{Acknowledgements}

This work was supported by the National Research Foundation of Korea (NRF), grant funded by the Korea government (MSIT) (2019R1F1A1041944), and the Radiation Technology R\&D program through the National Research Foundation of Korea funded by the Ministry of Science and ICT (2019M2A2B4095126).

\section{Conflicts of Interest}

The authors have nothing to disclose.

\section{Availability of Data and Materials}

All relevant data are within the paper and its Supporting Information files.

\section{Author Contributions}

Conceptualization: Minsoo Chun, Jung-in Kim. Data curation and formal analysis: Jeongho Kim, Jiwon Sung. Funding acquisition: Minsoo Chun, Chang Heon Choi. Investigation: Jiwon Sung, Minsoo Chun. Methodology: Jiwon Sung, Hyeongmin Jin, Minsoo Chun. Supervision: Minsoo Chun, Jong Min Park, Jung-in Kim. Validation: Jiwon Sung, Jeongho Kim. Writing-original draft: Jiwon Sung. Writingreview \& editing: Minsoo Chun.

\section{References}

1. Ma CMC, Chetty IJ, Deng J, Faddegon B, Jiang SB, Li J, et al. Beam modeling and beam model commissioning for Monte Carlo dose calculation-based radiation therapy treatment planning: Report of AAPM Task Group 157. Med Phys. 2020;47:el-e18.

2. Wanklyn MD, Kidane G, Crees L. Verification measurements of an eMC algorithm using a 2D ion chamber array. J Appl Clin Med Phys. 2016;17:320-328.

3. Chetty IJ, Curran B, Cygler JE, DeMarco JJ, Ezzell G, Faddegon BA, et al. Report of the AAPM Task Group No. 105: issues associated with clinical implementation of Monte Carlo-based photon and electron external beam treatment planning. Med Phys. 2007;34:4818-4853.

4. Pemler P, Besserer J, Schneider U, Neuenschwander H. Evaluation of a commercial electron treatment planning system based on Monte Carlo techniques (eMC). Z Med Phys. 2006;16:313-329.

5. Fix MK, Frei D, Volken W, Neuenschwander H, Born EJ, Manser P. Monte Carlo dose calculation improvements for low energy electron beams using eMC. Phys Med Biol. 2010;55:4577-4588.

6. Cygler JE, Daskalov GM, Chan GH, Ding GX. Evaluation of the first commercial Monte Carlo dose calculation engine for electron beam treatment planning. Med Phys. 2004;31: 142-153.

7. Xu Z, Walsh SE, Telivala TP, Meek AG, Yang G. Evaluation of the eclipse electron Monte Carlo dose calculation for small fields. J Appl Clin Med Phys. 2009;10:75-85.

8. Ding GX, Duggan DM, Coffey CW, Shokrani P, Cygler JE. First macro Monte Carlo based commercial dose calculation module for electron beam treatment planning--new issues for clinical consideration. Phys Med Biol. 2006;51: 2781-2799.

9. Neuenschwander H, Born EJ. A macro Monte Carlo method for electron beam dose calculations. Phys Med Biol. 1992; $37: 107-125$

10. Zhang A, Wen N, Nurushev T, Burmeister J, Chetty IJ. Comprehensive evaluation and clinical implementation of commercially available Monte Carlo dose calculation algorithm. J Appl Clin Med Phys. 2013;14:127-145.

11. Chamberland E, Beaulieu L, Lachance B. Evaluation of an electron Monte Carlo dose calculation algorithm for treatment planning. J Appl Clin Med Phys. 2015;16:60-79.

12. Łukomska S, Kukołowicz P, Zawadzka A, Gruda M, Giżyńska M, Jankowska A, et al. Evaluation of the usefulness of the electron Monte Carlo algorithm for planning radiotherapy with the use of electron beams. Pol J Med Phys Eng. 2016;22:49-54.

13. Jong WL, Ung NM, Tiong AHL, Rosenfeld AB, Wong JHD. Characterisation of a MOSFET-based detector for dose measurement under megavoltage electron beam radiotherapy. Radiat Phys Chem. 2018;144:76-84.

14. Fix MK, Cygler J, Frei D, Volken W, Neuenschwander H, Born EJ, et al. Generalized eMC implementation for Monte Carlo dose calculation of electron beams from different 
machine types. Phys Med Biol. 2013;58:2841-2859.

15. Ojala J, Kapanen M, Hyödynmaa S. Full Monte Carlo and measurement-based overall performance assessment of improved clinical implementation of eMC algorithm with emphasis on lower energy range. Phys Med. 2016;32:801811.

16. Varian Medical Systems. Eclipse photon and electron algorithms reference guide. Palo Alto: Varian Medical Systems; 2015.

17. Shimozato T, Kojima T, Sakamoto M, Hata Y, Sasaki K, Araki N. Evaluating the output stability of LINAC with a reference detector using 3D water phantom. Igaku Butsuri. 2012;32:176-181.
18. Khan FM, Gibbons JP. Khan's the physics of radiation therapy. Philadelphia: Lippincott Williams \& Wilkins; 2014.

19. Brualla L, Palanco-Zamora R, Wittig A, Sempau J, Sauerwein W. Comparison between PENELOPE and electron Monte Carlo simulations of electron fields used in the treatment of conjunctival lymphoma. Phys Med Biol. 2009; 54:5469-5481.

20. Edimo P, Kwato Njock MG, Vynckier S. Validation of XiO Electron Monte Carlo-based calculations by measurements in a homogeneous phantom and by EGSnrc calculations in a heterogeneous phantom. Phys Med. 2013;29:631638. 